\title{
Effect of Elastic Deformations on the Critical Behavior of Three-Dimensional Systems with Long-Range Interaction
}

\author{
S.V. Belin* \\ Omsk State University, 55-a, pr. Mira, Omsk, Russia, 644077 \\
}

(Dated: June 18, 2018)

\begin{abstract}
A field-theoretical description of the behavior of compressible Ising systems with long-range interactions is presented. The description is performed in the two-loop approximation in three dimensions with the use of the PadBorel resummation technique. The renormalization group equations are analyzed, and the fixed points that determine the critical behavior of the system are found. It is shown that the effect of elastic deformations on a system with a long-range interaction causes changes in its critical, as well as multicritical, behavior.
\end{abstract}

PACS numbers: 64.60.-i

In compressible systems, the relation of the order parameter to elastic deformations plays an important role. Earlier [1] it was shown that, in the case of an elastically isotropic body, the critical behavior of a compressible system with a quadratic striction is unstable with respect to the relation of the order parameter to acoustic modes, and a first-order phase transition close to a second-order one is realized. However, the conclusions formulated in the cited paper [1] hold only for low pressures. As was shown later [2], at high pressures beginning from a certain tricritical point $P_{t}$, the deformations induced by the external pressure affect the system to a greater extent and lead to a change of sign of the effective interaction constant for the order parameter fluctuations and, as a consequence, to a change in the order of the phase transition. In this case, according to [2], a homogeneous compressible system is characterized by two types of tricritical behavior with a fourth-order critical point formed as the point of intersection of the two tricritical curves. Calculations performed in terms of the two-loop approximation [3] confirmed the presence of two types of tricritical behavior for Ising systems and provided values of the tricritical indices.

In structural phase transitions that occur in the absence of the piezoelectric effect, in the paraphase the elastic strains play the role of a secondary order parameter whose fluctuations are not critical in most cases.

The effect of the long-range interaction described by the power law $1 / r^{-D-\sigma}$ at long distances was studied analytically in terms of the $\varepsilon$-expansion [46] and numerically by the Monte Carlo method [79] in two and three dimensions. It was found that the long-range interaction considerably affects the critical behavior of Ising systems for the parameter values $\sigma<2$. A recent study carried out for a three-dimensional space in the two-loop approximation [10] confirmed the prediction of the $\varepsilon$-expansion for systems with long-range interactions.

This paper describes the critical and tricritical behavior of three-dimensional compressible systems by taking

*Electronic address: belim@univer.omsk.su into account the effect of the long-range interaction with different values of the parameter $\sigma$.

For a homogeneous Ising-like model with elastic deformations and a long-range interaction, the Hamiltonian can be represented in the form

$$
\begin{aligned}
& H_{0}=\int d^{D} x\left[\frac{1}{2}\left(\tau_{1}+\nabla^{\sigma}\right) \vec{S}(x)^{2}++\frac{u_{0}}{4 !}\left(\vec{S}(x)^{2}\right)^{2}\right] \\
& +\int d^{D} x\left[a_{1}\left(\sum_{\alpha=1}^{3} u_{\alpha \alpha}(x)\right)^{2}+a_{2} \sum_{\alpha, \beta=1}^{3} u_{\alpha \beta}^{2}\right]+ \\
& +\frac{1}{2} a_{3} \int d^{D} x \vec{S}(x)^{2}\left(\sum_{\alpha=1}^{3} u_{\alpha \alpha}(x)\right)
\end{aligned}
$$

where $S(x)$ is the scalar order parameter, $u_{0}$ is a positive constant, $\tau_{0} \sim\left|T-T_{c}\right|, T_{c}$ is the phase transition temperature, $u_{\alpha \beta}$ is the strain tensor, $a_{1}$ and $a_{2}$ are the elastic constants of the crystal, and $a_{3}$ is the quadratic striction parameter. Let us change to the Fourier transforms of the variables in Eq. (1) and perform integration with respect to the components depending on the nonfluctuating variables, which do not interact with the order parameter $S(x)$ Then, introducing, for convenience, the new variable $y(x)=\sum_{\alpha=1}^{3} u_{\alpha \alpha}(x)$, we obtain the Hamiltonian of the system in the form

$$
\begin{aligned}
& H_{0}=\frac{1}{2} \int d^{D} q\left(\tau_{0}+q^{\sigma}\right) S_{q} S_{-q} \\
& +\frac{u_{0}}{4 !} \int d^{D} q_{i} S_{q 1} S_{q 2} S_{q 3} S_{-q 1-q 2-q 3}+ \\
& +a_{3} \int d^{D} q y_{q 1} S_{q 2} S_{-q 1-q 2}+\frac{a_{3}^{(0)}}{\Omega} y_{0} \int d^{D} q S_{q} S_{-q} \\
& +\frac{1}{2} a_{1} \int d^{D} q_{q} y_{-q}+\frac{1}{2} \frac{a_{1}^{(0)}}{\Omega} y_{0}^{2}
\end{aligned}
$$

In Eq. (2), the components $y_{0}$ describing the homogeneous strains are separated. According to [1], such a separation is necessary, because the inhomogeneous strains $y_{q}$ are responsible for the acoustic phonon exchange and 
lead to long-range interactions, which are absent in the case of homogeneous strains. For the system under study, let us determine the effective Hamiltonian that depends only on the strongly fluctuating order parameter $\mathrm{S}$

$$
\exp \{-H[S]\}=B \int \exp \left\{-H_{R}[S, y]\right\} \prod d y_{q}
$$

If the experiment is performed at constant volume, $y_{0}$ is a constant and the integration in Eq. (3) is performed with respect to only the inhomogeneous strains, while the homogeneous strains do not contribute to the effective Hamiltonian. If the experiment occurs at constant pressure, the term $P \Omega$ is added to the Hamiltonian, the volume is represented in terms of the strain tensor components as

$$
\Omega=\Omega_{0}\left[1+\sum_{\alpha=1} u_{\alpha \alpha}+\sum_{\alpha \neq \beta} u_{\alpha \alpha} u_{\beta \beta}+O\left(u^{3}\right)\right]
$$

and the integration in Eq. (3) is performed with respect to the homogeneous strains as well. According to [11], the inclusion of quadratic terms in Eq. (4) may be important when dealing with high pressures and crystals with large striction effects. The neglect of these quadratic terms restricts the applicability of the results obtained by Larkin and Pikin [1] to the case of low pressures. Thus, the Hamiltonian has the form

$$
\begin{aligned}
& H=\frac{1}{2} \int d^{D} q\left(\tau_{0}+q^{\sigma}\right) S_{q} S_{-q} \\
& +\left(u_{0}-\frac{z_{0}}{2}\right) \int d^{D} q_{1} d^{D} q_{2} d^{D} q_{3} S_{q 1} S_{q 2} S_{q 3}^{a} S_{-q 1-q 2-q 3} \\
& +\frac{1}{2 \Omega}\left(z_{0}-w_{0}\right) \int d^{D} q_{1} d^{D} q_{2} S_{q 1} S_{-q 1} S_{q 2} S_{-q 2} \\
& z_{0}=a_{1}^{2} /\left(4 a_{3}\right), \quad w_{0}=a_{1}^{(0) 2} /\left(4 a_{3}^{(0)}\right)
\end{aligned}
$$

The effective interaction parameter $v_{0}=u_{0}-z_{0} / 2$ that appears in the Hamiltonian due to striction effects, which are determined by the parameter $z_{0}$, can take not only positive but also negative values. As a result, the Hamiltonian describes both first-order and secondorder phase transitions. At $v_{0}=0$, the system exhibits a tricritical behavior. In its turn, the effective interaction determined in Hamiltonian (5) by the parameter difference $z_{0} w_{0}$ may cause a second-order phase transition in the system when $z_{0} w_{0}>0$ and a first-order phase transition when $z_{0} w_{0}<0$. This form of the effective Hamiltonian suggests that a higher order critical point can be realized in the system as the point of intersection of the tricritical curves when the conditions $v_{0}=0$ and $z_{0}=w_{0}$ are simultaneously satisfied [2]. It should be noted that, with the tricritical condition $z_{0}=w_{0}$, Hamiltonian (5) of the model under consideration is isomorphic with the Hamiltonian of a rigid homogeneous system.

In the framework of the field-theoretical approach [12], the asymptotic critical behavior and the structure of the phase diagrams in the fluctuation region are determined by the CallanSymanzik renormalization group equation for the vertex parts of the irreducible Green functions. To calculate the $\beta$ - and $\gamma$-functions as the functions involved in the CallanSymanzik equation for renormalized interaction vertices $u, a_{1}$, and $\left.a_{1}^{(} 0\right)$, or complex vertices $z=a_{1}^{2} / 4 a_{3}, w=a_{1}^{(0) 2} / 4 a_{3}^{(0)}$, and $v=u 12 z$, which are more convenient for the determination of critical and tricritical behavior, a standard method based on the Feynman diagram technique and on the renormalization procedure [13] was used with the propagator $G(\vec{k})=1 / \tau+|\vec{k}|^{\sigma}$. As a result, the following expressions were obtained for the $\beta$ - and $\gamma$-functions in the two-loop approximation:

$$
\begin{aligned}
& \beta_{v}=-(2 \sigma-D) v\left[1-36 v J_{0}+1728\left(2 J_{1}-J_{0}^{2}-\frac{2}{9} G\right) v^{2}\right], \\
& \beta_{z}=-(2 \sigma-D) z\left[1-24 v J_{0}-2 z J_{0}\right. \\
& \left.+576\left(2 J_{1}-J_{0}^{2}-\frac{2}{3} G\right) v^{2}\right] \\
& \beta_{w}=-(2 \sigma-D) w\left[1-24 v J_{0}-4 z J_{0}+2 w J_{0}\right. \\
& \left.+576\left(2 J_{1}-J_{0}^{2}-\frac{2}{3} G\right) v^{2}\right] \text {. } \\
& \gamma_{t}=(2 \sigma-D)\left[-12 v J_{0}-2 z J_{0}+2 w J_{0}\right. \\
& \left.+288\left(2 J_{1}-J_{0}^{2}-\frac{1}{3} G\right) v^{2}\right] \\
& \gamma_{\varphi}=(2 \sigma-D) 192 G v^{2} \\
& J_{1}=\int \frac{d^{D} q d^{D} p}{\left(1+|\vec{q}|^{\sigma}\right)^{2}\left(1+|\vec{p}|^{\sigma}\right)\left(1+\left|q^{2}+p^{2}+2 \vec{p} \vec{q}\right|^{\sigma / 2}\right)}, \\
& J_{0}=\int \frac{d^{D} q}{\left(1+|\vec{q}|^{\sigma}\right)^{2}} \text {, } \\
& G=-\frac{\partial}{\partial|\vec{k}|^{\sigma}} \int d^{D} q d^{D} p\left(1+\left|q^{2}+k^{2}+2 \vec{k} \vec{q}\right|^{\sigma}\right)^{-1} \\
& \left(1+|\vec{p}|^{\sigma}\right)^{-1}\left(1+\left|q^{2}+p^{2}+2 \vec{p} \vec{q}\right|^{\sigma / 2}\right)^{-1}
\end{aligned}
$$

Redefining the effective interaction vertices as

$$
v_{1}=v \cdot J_{0}, \quad v_{2}=z \cdot J_{0}, \quad v_{3}=w \cdot J_{0} .
$$

we arrive at the following expressions for the $\beta$ - and $\gamma$ - 
functions:

$$
\begin{aligned}
\beta_{1}= & -(2 \sigma-D) v_{1}\left[1-36 v_{1}\right. \\
& \left.+1728\left(2 \widetilde{J_{1}}-1-\frac{2}{9} \widetilde{G}\right) v_{1}^{2}\right], \\
\beta_{2}= & -(2 \sigma-D) v_{2}\left[1-24 v_{1}-2 v_{2}\right. \\
& \left.+576\left(2 \widetilde{J_{1}}-1-\frac{2}{3} \widetilde{G}\right) v_{1}^{2}\right], \\
\beta_{3}= & -(2 \sigma-D) v_{3}\left[1-24 v_{1}-4 v_{2}+2 v_{3}\right. \\
& \left.+576\left(2 \widetilde{J_{1}}-1-\frac{2}{3} \widetilde{G}\right) v_{1}^{2}\right], \\
\gamma_{t}= & (2 \sigma-D)\left[-12 v_{1}-2 v_{2}+2 v_{3}\right. \\
& \left.+288\left(2 \widetilde{J_{1}}-1-\frac{1}{3} \widetilde{G}\right) v_{1}^{2}\right], \\
\gamma_{\varphi}= & (2 \sigma-D) 192 \widetilde{G} v_{1}^{2} .
\end{aligned}
$$

The redefining procedure makes sense for a $\sigma \leq D / 2$. In this case, $J_{0}, J_{1}$, and $G$ become divergent functions. Introducing the cutoff parameter $\Lambda$ and considering the ratios $J_{1} / J_{0}^{2}, G / J_{0}^{2}$ in the limit of $\Lambda \rightarrow \infty$, we obtain finite expressions.

The values of the integrals were determined numerically. For the case a $a \leq D / 2$, a sequence of the values of $J_{1} / J_{0}^{2}$ and $G / J_{0}^{2}$ was constructed for different $\Lambda$ and then approximated to infinity.

It is well known that perturbative series expansions are asymptotic and the interaction vertices of the order parameter fluctuations in the fluctuation region are sufficiently large to directly apply Eqs. (7). Therefore, to extract the necessary physical information from the expressions derived above, the PadeBorel method generalized to the three-parameter case was used. The corresponding direct and inverse Borel transformations have the form

$$
\begin{aligned}
& f\left(v_{1}, v_{2}, v_{3}\right)=\sum_{i_{1}, i_{2}, i_{3}} c_{i_{1}, i_{2}, i_{3}} v_{1}^{i_{1}} v_{2}^{i_{2}} v_{3}^{i_{3}} \\
& =\int_{0}^{\infty} e^{-t} F\left(v_{1} t, v_{2} t, v_{3} t, v_{4} t\right) d t, \\
& F\left(v_{1}, v_{2}, v_{3}\right)=\sum_{i_{1}, i_{2}, i_{3}} \frac{c_{i_{1}, i_{2}, i_{3}}}{\left(i_{1}+i_{2}+i_{3}\right) !} v_{1}^{i_{1}} v_{2}^{i_{2}} v_{3}^{i_{3}} .
\end{aligned}
$$

For an analytical continuation of the Borel transform of a function, a series in an auxiliary variable is introduced:

$\tilde{F}\left(v_{1}, v_{2}, v_{3}, \theta\right)=\sum_{k=0}^{\infty} \theta^{k} \sum_{i_{1}, i_{2}, i_{3}} \frac{c_{i_{1}, i_{2}, i_{3}}}{k !} v_{1}^{i_{1}} v_{2}^{i_{2}} v_{3}^{i_{3}} \delta_{i_{1}+i_{2}+i_{3}, k}$

and the $[L / M]$ Pade approximation is applied to this series at the point $\theta=1$. This approach was proposed and tested in [14] in describing the critical behavior of systems characterized by several vertices of interaction of the order parameter fluctuations. The property [14] of the system retaining its symmetry under the Pade approximants in the variable is essential in the description of multivertex models.
In the two-loop approximation, the functions were calculated using the [2/1] approximant. The character of the critical behavior is determined by the existence of a stable fixed point satisfying the set of equations

$$
\beta_{i}\left(v_{1}^{*}, v_{2}^{*}, v_{3}^{*}\right)=0 \quad(i=1,2,3) .
$$

The requirement that the fixed point be stable is reduced to the condition that the eigenvalues $b_{i}$ of the matrix

$$
B_{i, j}=\frac{\partial \beta_{i}\left(v_{1}^{*}, v_{2}^{*}, v_{3}^{*}\right)}{\partial v_{j}} .
$$

lie in the half-plane of the right-hand complex. The fixed point with $v^{*}=0$, which corresponds to the tricritical behavior, is a saddle point and must be stable in the directions determined by the variables $z$ and $w$ and unstable in the direction determined by the variable $v$. The stabilization of the tricritical fixed point in the direction determined by the variable $v$ is achieved by taking into account the sixth-order terms with respect to the order parameter fluctuations in the effective Hamiltonian of the model. The fixed point with $z^{*}=w^{*}$, which corresponds to the tricritical behavior of the second type, is also a saddle point and must be stable in the directions determined by the variables $v$ and $z$ and unstable in the direction determined by the variable $w$. Its stabilization is possible at the expense of the anharmonic effects.

The resulting set of the resummed $\beta$ functions contains a wide variety of fixed points. The table specifies the fixed points that are of most interest for describing the critical and tricritical behavior and lie in the physical region of vertex values with $v, z, w \geq 0$. The table also shows the eigenvalues of the stability matrix for the corresponding fixed points and the critical indices $\nu$ and $\eta$.

The analysis of the values and stability of the critical points suggests the following conclusions. Qualitatively, the critical phenomena seem to be identical for any value of the long-range interaction parameter $a$. The critical behavior of incompressible systems is unstable with respect to the deformation degrees of freedom (points 1). The stable point proves to be the one at a constant strain (points 2). Fixed points 3 describe the first type of tricritical behavior of compressible systems, which occurs at constant pressure. Fixed points 4 are tricritical for systems studied at constant volume. Points 5 are fourthorder critical points at which two tricritical curves intersect.

For the tricritical behavior of the first type (points 3), Hamiltonian (5) is isomorphic with the Hamiltonian of an incompressible homogeneous model and, hence, the critical indices also coincide with those of the incompressible model. The tricritical behavior of the second type (points 4) corresponds to the critical behavior of a spherical model and is determined by the corresponding indices. The fourth-order fixed points (points 5) are characterized by the field-average values of the critical indices. 
The large values of the effective vertices $z$ and $w$ in comparison with the systems with short-range interactions [3] are caused by the fact that the mechanism governing the effect of elastic deformations on the critical phenomena is related to the dependence of the interaction integral in the Ising model on the distance between the lattice sites.

The study described above revealed the considerable effect of elastic deformations on the critical behavior of systems with a long-range interaction. This effect manifests itself as a change in the values of the critical indices for Ising systems along with the appearance of multicritical points in the phase diagrams of the substances.

The work is supported by Russian Foundation for Basic Research N 04-02-16002.
[1] A. I. Larkin and S. A. Pikin, Zh. ksp. Teor. Fiz. 56, 1664 (1969) [Sov. Phys. JETP 29, 891 (1969)].

[2] Y. Imry, Phys. Rev. Lett. 33 (21), 1304 (1974).

[3] S. V. Belim and V. V. Prudnikov, Fiz. Tverd. Tela (St. Petersburg) 43, 1299 (2001) [Phys. Solid State 43, 1353 (2001)].

[4] M. E. Fisher, S.-K. Ma, and B. G. Nickel, Phys. Rev. Lett. 29, 917 (1972).

[5] J. Honkonen, J. Phys. A 23, 825 (1990).

[6] E. Luijten and H. Mebingfeld, Phys. Rev. Lett. 86, 5305 (2001).

[7] E. Bayong and H. T. Diep, Phys. Rev. B 59, 11919 (1999).

[8] E. Luijten, Phys. Rev. E 60, 7558 (1999).
[9] E. Luijten and H. W. J. Blote, Phys. Rev. B 56, 8945 (1997).

[10] S. V. Belim, Pisma Zh. ksp. Teor. Fiz. 77, 118 (2003) [JETP Lett. 77, 112 (2003)].

[11] D. J. Bergman and B. I. Halperin, Phys. Rev. B 13, 2145 (1976).

[12] D. Amit, Field Theory the Renormalization Group and Critical Phenomena (McGraw-Hill, New York, 1976).

[13] J. Zinn-Justin, Quantum Field Theory and Critical Phenomena (Clarendon Press, Oxford, 1989).

[14] A. I. Sokolov and K. B. Varnashev, Phys. Rev. B 59, 8363 (1999). 


\begin{tabular}{|c|c|c|c|c|c|c|c|c|}
\hline $\mathrm{N}$ & $v_{1}^{*}$ & $v_{2}^{*}$ & $v_{3}^{*}$ & $b_{1}$ & $b_{2}$ & $b_{3}$ & $\nu$ & $\eta$ \\
\hline \multicolumn{7}{|c|}{$\sigma=1.9$} \\
\hline 1 & 0.042067 & 0 & 0 & 0.684 & -0.184 & -0.184 & 0.652688 & 0.113420 \\
2 & 0.044353 & 0.095190 & 0 & 0.684 & 0.185 & 0.183 & 0.751433 & 0.113420 \\
3 & 0.044353 & 0.095190 & 0.095190 & 0.684 & 0.185 & -0.185 & 0.652688 & 0.113420 \\
4 & 0 & 0.5 & 0 & -1 & 1 & 1 & 1.052632 & 0.1 \\
5 & 0 & 0.5 & 0.5 & -1 & 1 & -1 & 0.526316 & 0.1 \\
\hline \multicolumn{7}{|c|}{$\sigma=1.8$} \\
\hline 1 & 0.023230 & 0 & 0 & 0.628 & -0.488 & -0.488 & 0.636349 & 0.207461 \\
2 & 0.023230 & 0.245404 & 0 & 0.628 & 0.489 & 0.490 & 0.782912 & 0.207461 \\
3 & 0.023230 & 0.245404 & 0.245404 & 0.628 & 0.489 & -0.489 & 0.636349 & 0.207461 \\
4 & 0 & 0.5 & 0 & -1 & 1 & 1 & 1.111111 & 0.2 \\
5 & 0 & 0.5 & 0.5 & -1 & 1 & -1 & 0.555556 & 0.2 \\
\hline \multicolumn{7}{|c|}{$\sigma=1.7$} \\
\hline 1 & 0.020485 & 0 & 0 & 0.699 & -0.532 & -0.532 & 0.667452 & 0.304862 \\
2 & 0.020485 & 0.266497 & 0 & 0.699 & 0.533 & 0.532 & 0.830648 & 0.304862 \\
3 & 0.020485 & 0.266497 & 0.266497 & 0.699 & 0.533 & -0.533 & 0.667452 & 0.304862 \\
4 & 0 & 0.5 & 0 & -1 & 1 & 1 & 1.176471 & 0.3 \\
5 & 0 & 0.5 & 0.5 & -1 & 1 & -1 & 0.588235 & 0.3 \\
\hline \multicolumn{7}{|c|}{$\sigma=1.6$} \\
\hline 1 & 0.015974 & 0 & 0 & 0.874 & -0.616 & -0.616 & 0.697361 & 0.403936 \\
2 & 0.015974 & 0.309684 & 0 & 0.874 & 0.617 & 0.620 & 0.889473 & 0.403936 \\
3 & 0.015974 & 0.309684 & 0.309684 & 0.874 & 0.617 & -0.618 & 0.697361 & 0.403936 \\
4 & 0 & 0.5 & 0 & -1 & 1 & 1 & 1.25 & 0.4 \\
5 & 0 & 0.5 & 0.5 & -1 & 1 & -1 & 0.625 & 0.4 \\
\hline
\end{tabular}

